\title{
Presencias, sensibilidades y políticas cotidianas del habitar en el Atrato
}

\author{
Pilar Riaño-Alcalá y Natalia Quiceno Toro
}

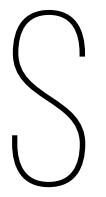

eguir el Atrato y las relaciones que lo componen nos acerca a sus ritmos, flujos y remolinos, así como a cambios en su cauce, bloqueos de diversa naturaleza, sedimentaciones y peces envenenados. Acercarse a sus sonidos, subidas y bajadas del caudal, a la red que hace con más de 15 ríos afluentes y 300 quebradas, muestra una cuenca de gran magnitud y profunda riqueza. La vida del río es también la vida de muchos seres y entornos que son parte de él. Declarado sujeto de derechos por la Sentencia T-622 del 2016¹, este lugar geohistórico configura mundos de vida humanos y no humanos, gentes y pueblos, ecologías y paisajes singulares. En este número especial pensamos con y desde el río Atrato para rastrear cómo este complejo entramado de historias, materias, seres y relaciones fluidas genera un repertorio creativo de acciones políticas de parte de sus habitantes, que responden a la muerte, al despojo y los desequilibros producidos en la historia más reciente del conflicto armado y de extracción voraz de sus recursos, así como a la larga historia de despojo, desplazamiento y destrucción ligada al colonialismo y al desarrollo capitalista en la región.

El Atrato fluye de sur a norte hasta desembocar en el golfo de Urabá, donde se encuentra con el mar Caribe a través de 16 bocas que crean un gran delta. Con sus $750 \mathrm{~km}$ de recorrido por el Chocó biogeográfico, de los cuales 500 son navegables, este cuerpo de agua pone en comunicación una inmensa red de ríos y quebradas, pero también sistemas montañosos, ya que la cuenca se encuentra en medio de la cordillera Occidental, la serranía del Baudó y el Darién. El Atrato circula por una de las regiones más biodiversas del mundo, es el río más caudaloso de Colombia y se localiza en la región que tiene los niveles de precipitaciones más elevados del continente. Todas estas singularidades hacen de la cuenca un

Esta sentencia de la Corte Constitucional es fruto de la articulación de organizaciones y autoridades étnicas de la región con el Centro de Estudios para la Justicia Social Tierra Digna, quienes demandaron a 26 entidades del Estado mediante una acción de tutela que buscaba garantías para los derechos fundamentales de las poblaciones asentadas en la cuenca. El fallo histórico reconoce al río como una entidad viviente que sostiene otras formas de vida, por lo tanto, es un sujeto de especial protección y con derecho a la conservación, mantenimiento y restauración. En este sentido, reparar los daños del río significa igualmente garantizar los derechos de las comunidades que habitan la cuenca. Para ampliar, véase https://tierradigna. org/pdfs/SomosGuardianesDelAtrato.pdf 
lugar de gran riqueza biológica, histórica y cultural. En este entorno diverso y caudaloso también se sufren amenazas y daños, particularmente por la contaminación y deforestación asociadas a procesos extractivos como la minería con dragones y dragas, la extracción maderera, y los controles territoriales y de movimiento por parte de guerrillas, paramilitares y ejército que hacen/deshacen los flujos de vida en el Atrato.

El trabajo antropológico sobre el Atrato que presentamos propone e invita a pensar sobre los modos como se configuran las vidas en ecologías de múltiples agentes, donde no solo se trata de una dependencia de la gente con el río, sino de una cocreación e interdependencia que forma unos modos de vivir en constante transformación y tensión. Respondemos así al llamado que Camargo y Camacho (2018) hicieron en un número anterior de la Revista Colombiana de Antropología acerca de la poca atención que los cuerpos de agua dulce han recibido en la disciplina y el amplio campo investigativo y teórico que se abre cuando pensamos e investigamos con los ríos, sus cuencas y corrientes. En esta introducción indagamos por las formas del río Atrato en relación con otros desde las pistas etnográficas que brindan las diferentes contribuciones. También es una invitación a embarcarse en este número especial de la $R C A$ para pensar con el Atrato los legados ecológicos, materiales, espirituales, sociales y culturales que ha dejado la guerra durante los últimos cuarenta años en la región.

$\mathrm{El}$ agua como un cuerpo en constante movimiento y sustancia que compone nuestros cuerpos provee un referente tangible y simbólico para resaltar la fluidez de las relaciones que un río como el Atrato teje o activa, el caudal narrativo de la multitud de seres que se relacionan con él y los flujos continuos que marcan el quehacer del río y de los seres que vivifica (Christian y Wong 2016; Krause y Strang 2013). Al trabajar en este número temas tan diversos como las plantas, los tejidos, la muerte, los cantos de alabaos, las exhumaciones, los consejos comunitarios y la lucha por los territorios que tienen en el río Atrato su referente, el entramado conceptual que guía nuestras reflexiones sobre la acción política y los legados de la guerra adquiere fuerza y movimiento. Acudimos a las nociones de presencias, sensibilidades y políticas cotidianas, que desarrollamos más adelante, para nombrar los encuentros, movimientos y relaciones con el río así como las posibilidades de acción que otorgan.

Estos movimientos y cauces de la guerra necesariamente se incrustan en las profundidades históricas de los pueblos indígenas que ancestralmente lo han habitado y navegado, de los hombres y mujeres que por sus aguas escaparon de la esclavitud o encontraron dónde asentarse una vez lograron la libertad. Historias de navegación, desplazamiento, trabajo forzado y violencias de todo tipo 
han activado prácticas y políticas cotidianas del habitar en el Atrato para sostener la vida y los mundos de seres que cohabitan allí. ¿Qué hacer con la violencia masiva que distorsiona los entramados de relaciones?, ¿cómo enfrentar los ciclos de agresión-violencia que retornan mientras los atrateños intentan imaginar y construir la paz?, ¿cómo habitar con los muertos que no logran obtener descanso y con los modos en que la guerra y la extracción incontrolada ensucian sus aguas?, ¿qué modos de hacer política exige restaurar la vida del río y de todos aquellos que lo producen y son producidos con él?

Como bien lo muestran Camargo y Camacho (2018), "las múltiples imaginaciones de los ríos y el agua en general enriquecen el debate político y académico, a la vez que dan pie a nuevas tensiones epistemológicas y ontológicas” (17). De estas tensiones dan cuenta varios de los artículos sobre el Medio y Bajo Atrato que documentan las amenazas al conocimiento y los modos de vivir con el río como eje de la vida y de las relaciones de los pueblos afros e indígenas, por la expansión de los proyectos extractivos y de control armado territorial y ribereño. Si bien las relaciones hidrosociales (Camacho y Camargo 2018) en el Atrato frente al cambio climático y frente a la minería extractiva (López-Gallego et al. 2017) no se abordan en este volumen, son un tema vigente en la cuenca, especialmente cuando se trata de indagar por los cambios en los ciclos de vida y las relaciones con el agua, las transformaciones en las subiendas de peces o los procesos de ordenamiento territorial ${ }^{2}$.

Como lo señala Lahiri-Dutt (2018), los modos en que son pensados, conceptualizados y ritualizados los ríos importan, puesto que de esas relaciones recíprocas y de interdependencia entre cuerpos y aguas emergen modos de existencia. Pensar esta dimensión en el Atrato invita a investigaciones más profundas sobre la rica tradición oral y literatura local que narran el río y los seres que lo habitan, así como sobre las prácticas rituales para su cuidado y sostenimiento, donde sus aguas son fundamentales para comprender los modos de ordenamiento propio y de relación entre los habitantes locales y el raudal. Pero también invita a profundizar en las maneras como el río ha sido imaginado y representado en los proyectos de desarrollo de la región pacífica, en las conexiones entre los océanos Atlántico y Pacífico, en los discursos que producen la noción de Chocó biogeográfico o en los planes e iniciativas de navegabilidad. Si entendemos entonces a los ríos como cuerpos de agua que están profundamente imbricados en el quehacer social, cultural y económico de una sociedad (Lahiri-Dutt 2018), los

2 Véase Majestuoso Atrato: relatos bioculturales del río. Reflexiones académicas y comunitarias de realidades y futuros del Chocó (2017), que documenta desde diversas perspectivas disciplinarias y comunitarias estas transformaciones. 
pensaremos también como cuerpos afectados por la guerra. Por ello, pensar con los ríos también invita a investigar desde una mirada integral y relacional la restitución de tierras, la reparación colectiva o el reconocimiento del territorio como víctima, en los actuales procesos de planeación con enfoque territorial que se están implementando en el marco de los acuerdos de paz.

Tres ejes de lectura nos permitirán trazar esas relaciones que hacen al Atrato: los seres que lo habitan, sus ecologías y los modos de hacer política. En el primero, nos guiamos por las presencias espectrales o materiales que hacen el lugar, que dan forma al espacio habitado y a los circuitos y el movimiento de seres e historias por sus aguas. El segundo eje tiene que ver con las sensibilidades, estéticas y conocimientos involucrados en la producción e imaginación política del río, como sujeto de derechos, como agente que hace posibles otras vidas. En este eje nos interesa cómo se nombran y se conceptualizan desde las perspectivas locales los problemas, legados, paisajes y ecologías propios de la cuenca en el presente. El tercer eje se enfoca en las políticas cotidianas del habitar, donde el trabajo del cuidado, la recomposición y los circuitos afectivos crean posibilidades de recuperar mundos y dar lugar a la reparación social.

\section{Presencias}

El término presencia describe la condición de alguien o de algo que se encuentra en un determinado lugar y lo que genera, incluyendo las sensaciones y las reacciones. Entre los legados que el conflicto armado deja en el Atrato, varios de los autores en esta edición indagan por las presencias espectrales o materiales. Daniel Ruiz-Serna examina el confinamiento de espíritus de paramilitares muertos en combate en el Bajo Atrato chocoano, su estado de abandono y olvido, y los encuentros afectivos con estas presencias de quienes retornaron a su territorio luego del desplazamiento. Camila Orjuela reflexiona sobre las presencias de los muertos de la masacre de Bojayá en los sueños de sus familiares y las cargas afectivas y deudas que generan cuando sus restos se encuentran perdidos o enterrados sin los rituales propios de los pueblos afrochocoanos. Pilar Riaño-Alcalá y Ricardo Chaparro indagan por las presencias acústicas en el Atrato: el registro afectivo y las resonancias de los alabaos, y las maneras en que lugares como la fosa donde sepultaron a los muertos de la masacre de Bojayá conservan la presencia afectiva y mnemónica de quienes ya no están enterrados allí. 
Estas presencias, como se puede concluir de la lectura de estos tres artículos, son tanto materiales como espectrales, tangibles e intangibles, y se perciben desde una variedad de registros afectivos. Es decir, tienen poder, resonancia y alcance sobre los seres humanos y no humanos, y sobre el mismo lugar. Daniel Ruiz-Serna muestra de qué modo los impulsos sutiles y las energías que emanan de las presencias espectrales de quienes sufrieron una mala muerte, así como las ruinas de un campamento abandonado, hacen y transforman tanto la experiencia del lugar como el mismo sentido de este para quienes lo han habitado por generaciones. Cuerpo y lugar, nos dice, son coconstituyentes de los afectos. Así como el río Atrato ejerce un poder sobre los seres humanos y no humanos, y genera ensamblajes complejos de relaciones espaciales entre seres vivos y el medio ambiente acuático (Oslender 2016), estas presencias espectrales y materiales provocan afectos y sensaciones que labran mundos de vida y muerte en el Atrato, tanto como las temporalidades y ciclos que los marcan.

La teoría de los afectos centra su atención en fuerzas viscerales, como estas presencias, que operan sigilosamente y en general por fuera o más allá del conocimiento consciente y que llevan hacia el movimiento, el pensamiento o la acción (Seigworth y Gregg 2010). Al reconocer estas fuerzas y presencias, la antropología de los afectos se interesa por la manera en que el medio ambiente y los objetos ejercen una fuerza generativa sobre los seres humanos, es decir, tienen capacidad agentiva propia y transmiten afectos y sensaciones en estos encuentros entre los seres y el entorno (Navarro-Yashin 2012). Esta interacción afectiva y su capacidad de generar acción entre lugares y seres humanos y no humanos está arraigada en los modos de ver el mundo y en el día a día de los pueblos afrochocoanos y emberas del Atrato (Flórez 2007; González y González 2017; Ulloa 1992). José de la Cruz Valencia, en su manuscrito para este número, evoca la Bojayá de su infancia y una convivencia en la que "toda la comunidad se movía alrededor del río Atrato”. Él rememora cómo la subienda daba pie a la celebración y a rituales cotidianos de agradecimiento al río por la abundancia. De la misma manera, la poeta sto:lo Lee Maracle, en el ensayo que se ha traducido para esta edición, escribe sobre el respeto mutuo y la humildad que establecen su nación y las demás naciones indígenas de la Costa Salish en Canadá con el agua y el río.

El reconocimiento de estas fuerzas afectivas ancla nuestra comprensión sobre la acción política porque hace visible la conexión entre el desequilibro y las ansiedades que ha generado la guerra en el Atrato, y el sentido y poder de un conjunto de acciones creativas con las que sus gentes responden a la violencia y a las dinámicas extractivistas. 


\section{Sensibilidades}

Fiestas religiosas emblemáticas como la celebración del santo patrono de Quibdó, san Pacho, expresiones como la chirimía y el alabao, y los cultivos y siembras en patios y azoteas relatan la riqueza histórica y cultural de esta región. Un conjunto de prácticas y saberes que también hacen al Atrato pero que en particular animan sus corrientes y los modos de habitar y navegar el río como lugar de ceremonia, fiesta, canto, danza, expresión artística y conocimiento ancestral afroindígena. En este número interrogamos las corrientes y tramas narrativas que circulan por el Atrato de hoy y las historias que cuentan y ponen en movimiento. Como veremos en algunos de los artículos, estas narrativas revelan sensibilidades peculiares, es decir, unas maneras de sentir y pensar, y unas capacidades de percepción que se forman en el encuentro con los ríos, los territorios y la selva -entre expresiones estéticas y rituales que conectan mundos de vida y muerte-, y también en los modos de sobrellevar el día a día y habitar los territorios en medio de la violencia y la guerra de las últimas décadas en la cuenca del Atrato.

Diego Cagüeñas, María Isabel Galindo y Sabina Rasmussen exploran las relaciones políticas que se ponen en juego ante el reconocimiento del Atrato como entidad viviente titular de derechos. La novedad de la Sentencia T-622 no reside únicamente en que es la primera de este tipo en Colombia y en el continente, sino además en las posibilidades que abre para recrear relaciones y maneras de cuidar, defender y sostener el río y sus afluentes. Para ello, los orilleros atrateños, actuando ahora como guardianes del Atrato, embarcan sus sensibilidades acuáticas y su capacidad de percibir las transformaciones del río, las aguasucias y el sentir de este cuerpo de agua para avanzar en un ejercicio inédito y colectivo de imaginación ecopolítica.

Las nociones de aguasucia y mala muerte que analizan varios de los artículos no se limitan a ser metáforas de las experiencias de vida y muerte en el Atrato. Hacen parte del repertorio local de nociones y conocimiento situado con el que se nombran y caracterizan los impactos devastadores de las violencias sobre la ecología, la vida y la muerte, y los recursos. Además, captan los efectos profundos en los modos de ser del río (el aguasucia) y los modos de sentir y sufrir de las personas, por ejemplo, el impacto de la muerte súbita, masiva y sin los cuidados rituales apropiados hacia el mismo territorio y los seres que lo habitan (Orjuela y Ruiz-Serna). Seguir las pistas de estos modos de nombrar y narrar permite a los autores registrar cómo se hace la política desde las epistemologías locales del daño. 
Unidos a estas sensibilidades o modos de percibir los efectos del entorno y el repertorio de acciones políticas y formas de narrar la vida y la violencia, se encuentran los procesos de reconstitución de las subjetividades individuales y colectivas de las mujeres y hombres que habitan la región. La subjetividad, argumentan Biehl, Good y Kleinman (2007), es un medio para darle forma a la sensibilidad. La noción de subjetividad como referente empírico y analítico alude al mundo interior de la experiencia sentida del sujeto. Esta experiencia es el resultado de las interacciones con las instituciones (incluyendo el Estado), la familia y la comunidad, de las memorias de las intervenciones coloniales, de las violencias continuas y las maneras como los sujetos viven y piensan su existencia (Biehl, Good y Kleinman 2007).

Natalia Quiceno Toro y Adriana Villamizar se enfocan en dos colectivos de mujeres rurales del Medio Atrato que actualizan sus saberes y reconstruyen sus vidas con el aprendizaje y trabajo colectivo con textiles. La reconfiguración creativa de sus espacios de vida, de los oficios y conocimientos y de las sensibilidades estéticas y políticas de sus prácticas, nos dicen las autoras, reconstituyen a la vez sus subjetividades. Por su parte, Bela Henríquez registra las prácticas de cultivo en patios y azoteas y las maneras creativas en las que siete mujeres bellavisteñas revitalizan el cultivo de plantas ornamentales y medicinales sembrándolas en andenes y en patios luego de la reubicación de su pueblo. Estos paisajes construidos bajo una intención estética y de cuidado de los otros transforman el espacio y desatan su imaginación creativa. La subjetividad de estas mujeres aflora en la sensibilidad estética del diseño y cultivo de las azoteas y patios, y en su capacidad de captar valores estéticos afrocolombianos y unas memorias ancestrales. Mediante este tipo de prácticas, ellas van fundamentando espacios de autonomía y recomposición como sujetos, y estos a la vez son reconstituyentes de las relaciones entre plantas, siembra, río, selva y mujeres. Este acercamiento a las subjetividades establece una continuidad entre la experiencia interna sentida (Das y Kleinman 2000) que moldea a la persona, sus modos de hacer y pensar, y la experiencia exteriorizante de su relación con otros sujetos y el entorno, con los lugares, espacios y presencias que están por fuera o más allá del sujeto (NavarroYashin 2012).

Por último, los artículos conectan este repertorio de sensibilidades, afectos y subjetividades con la prevalencia o el resurgimiento de unos modos de habitar el río y el territorio anclados en una ética del cuidado y el respeto. Ética y estética del cuidado afirman la dimensión política y las capacidades locales de imaginarse y recrear el quehacer de la política en el día a día. Lee Maracle alerta sobre las relaciones de carácter extractivo y destructivo con el agua y la tierra que han 
marcado la historia del colonialismo. Invita a pensar en modos de existencia y políticas del cuerpo que, fundamentados en una ética del cuidado y la responsabilidad, interpelen la idea de que el agua nos pertenece. El agua no nos pertenece ni puede ser gobernada, afirma, el agua es dueña de sí misma. En la tradición salish, las personas tienen la obligación de tener una "buena relación” con el agua y de ser humildes frente a ella.

\section{Políticas cotidianas del habitar}

En un reciente informe periodístico del diario El Colombiano y la Defensoría del Pueblo se destaca el lugar que han ganado la coca, y su cultivo, procesamiento y tráfico, en lo que denominan "el edén chocoano" "Guerra y coca: la maldición del edén chocoano” 2020). Las articulaciones entre guerra y coca afectan la región del litoral de este departamento, al tiempo que marcan nuevas dinámicas de violencia en ciudades como Quibdó, el mayor centro urbano de la cuenca del Atrato (con aproximadamente 150.000 habitantes). Miles de campesinos afros, mestizos e indígenas reconfiguraron el poblamiento de esta ciudad en la búsqueda de refugio en medio del desplazamiento forzado que vivió su peor crisis a finales de la década de 1990 (Abadía, en este número; Quiceno y Villamizar, en este número). Hoy estos habitantes se enfrentan a historias similares en el contexto urbano. La violencia en la región se debe leer también desde la ciudad que igualmente es el Atrato (Arango 2013) y de lo que nos dicen las cifras: 20 muertos en los dos primeros meses del 2020, 216 en el año anterior y 208 en el 2018, sin contar con el probable subregistro que se materializa en los cementerios clandestinos de los que se rumora como secretos a voces en la ciudad.

En Colombia los ríos son testigos no tan silenciosos de lo que ha sido la guerra. ¿Cómo se incorporan la historia de los acontecimientos y las dinámicas de violencia en las ecologías y relaciones vitales que componen el río Atrato? ¿Cómo se habitan esos paisajes? En este número vemos cómo alrededor de las plantas (Henríquez), los textiles (Quiceno y Villamizar) y los procesos de retorno (Abadía) se diseñan políticas cotidianas del habitar que contribuyen a la reparación social y ambiental. Por políticas cotidianas del habitar entendemos ese repertorio de acciones con las que se negocia y contesta a la desposesión y la violencia en la vida diaria, y cuyos sentidos no se agotan en la sobrevivencia o la respuesta directa sino que se labran en la compleja labor de reconstituir mundos y relaciones: en el recrear modos de habitar en entornos destruidos para que la vida 
florezca poco a poco, en la creatividad cotidiana y en la negociación de motivaciones, tensiones y deseos que acompañan las intervenciones institucionales en los territorios y su defensa. Centrarse en lo cotidiano es resaltar la relevancia de estas tensiones, así como los espacios en los que se revivifican lo interpersonal, lo social y la relación entre multiespecies a través de diversas prácticas cotidianas y de memoria (Riaño y Baines 2012).

Los artículos nos permiten entender que los daños sociales y ambientales pueden ser diferenciados pero son, ante todo, procesos interdependientes que dejan huellas particulares en la cuenca. En ese sentido, los trabajos etnográficos en el Medio y Bajo Atrato nos presentan una serie de prácticas, conocimientos y tecnologías comunitarias que han producido estrategias locales para emprender procesos reparadores de sus mundos socionaturales. Wisthon Andrés Abadía, en su trabajo con consejos comunitarios de la cuenca del Cacarica en el Bajo Atrato, describe las tensiones y disputas por la producción de sus territorios en relación con políticas de reconocimiento étnico, dinámicas de destierro, procesos de retorno y activismo desde los derechos humanos. Evidencia cómo esas prácticas cotidianas del habitar se van reconfigurando en medio de las dinámicas de despojo y restitución de derechos territoriales. Comprender que habitar la cuenca tiene relación con la violencia, la resistencia y la acción política nos exige estar atentos a los procesos socioespaciales articulados a la movilización social (Oslender 2008), así como a los procesos históricos que han configurado territorios vaciados como producto de órdenes raciales, coloniales y regímenes de desigualdad que perviven hasta el presente (Vergara-Figueroa 2018).

En este número se muestra cómo la producción del territorio atrateño también depende de los modos en que las violencias se van sedimentando, dando como resultado ríos que son fosas comunes, trabajaderos donde aparecen los espíritus de los muertos en combate, además de espacios de memoria para honrar a los muertos, recuperar la confianza y cultivar la dignidad. Sobre estos últimos espacios vemos que las resistencias y reexistencias se manifiestan en el lenguaje de las huertas y los bordados. Espacios emergentes que transforman las áridas políticas de reparación del Estado en lugares en los que se reconstruyen los vínculos entre personas, comunidades, plantas, memorias, agua y tierra.

El concepto de vida en el Atrato, escribió el antropólogo chocoano Rogerio Velásquez (2000), “es movimiento, acción, oportunidad de hacer” (140). Este modo de entender la vida revela también las maneras en que ciertas acciones y rituales adquieren un sentido reparador: los cantos de alabaos viajan por el río y crean un entorno afectivo que permite responder al exceso de muerte y pérdida (Riaño-Alcalá y Chaparro, en este número); las exhumaciones y el entierro permiten 
a los espíritus emprender el camino como ancestros, los rituales se reinventan y los ritmos temporales de las prácticas mortuorias se expanden para responder a la muerte masiva y que las ánimas lleguen "a puerto seguro" (Orjuela, en este número). Mujeres y hombres afros e indígenas de las diferentes orillas del río se convierten, con la Sentencia T-622, en "guardianes del Atrato" y se adentran en ejercicios creativos de imaginar, con las aguas de su río, los modos de cuidarlo, habitarlo y recrear sus identidades como orilleros guardianes (Cagüeñas, Galindo y Rasmussen). Estas son formas de la política centradas en la restitución de las relaciones entre personas (Baines 2016) y de las cualidades del lugar, el río y los seres que lo habitan.

\section{Referencias}

Arango Melo, Ana María. 2013. "La recuperación del espacio perdido: educación musical, adoctrinamiento y resistencia en Quibdó (Chocó-Colombia)”. Boletín del Observatorio del Patrimonio Culturaly Arqueológico (OPCA), número especial "La música como patrimonio, identidad y mestizaje” 5: 62-68.

Baines, Erin. 2016. Buried in the Heart: Women, Complex Victimhood and the War in Northern Uganda. Cambridge: Cambridge University Press.

Biehl, João, Byron Good y Arthur Kleinman. 2007. Subjectivity: Ethnographic Investigations, vol. 7. Berkeley: University of California Press.

Camargo, Alejandro y Juana Camacho. 2018. “Convivir con el agua”. Revista Colombiana de Antropología 55 (1): 7-25. https://doi.org/10.22380/2539472X.567

Christian, Dorothy y Rita Wong. 2016. Downstream: Reimagining Water. Waterloo, Ontario: Wilfrid Laurier University Press.

Das, Veena y Arthur Kleinman. 2000. Introducción a Violence and Subjectivity, editado por Veena Das, Arthur Kleinman, Manphela Ramphele y Pamela Reynolds, 1-19. Berkeley: University of California Press.

Flórez López, Jesús Alfonso. 2007. Autonomía indígena en Chocó. Medellín: Ediciones CEE.

González, Ximena y Viviana González. 2017. “Derechos bioculturales y derechos de los ríos: una interpelación al modelo minero energético en el departamento del Chocó”. En Majestuoso Atrato 2017.

“Guerra y coca: la maldición del edén chocoano”. 2020. El Colombiano. https://www.elcolombiano.com/reportajes/guerra-bandas-criminales-y-coca-en-choco

Krause, Franz y Veronica Strang. 2013. “Thinking Relationships Through Water”. Society \& Natural Resources 29 (6): 633-638. https://doi.org/10.1080/08941920.2016.1151714 
Lahiri-Dutt, Kuntala. 2018. "Imaginando los ríos”. Revista Colombiana de Antropología 55 (1): 153-166. https://doi.org/10.22380/2539472X.574

López-Gallego Cristina, Juan Felipe Blanco, Brian Bock, Luz Fernanda Jiménez, Vivian Páez y Juan Luis Parra. 2017. "El impacto de la destrucción y degradación de ecosistemas por minería, deforestación y explotación forestal en la biodiversidad del Chocó”. En $M a-$ jestuoso Atrato 2017.

Majestuoso Atrato: relatos bioculturales del río. Reflexiones académicas y comunitarias de realidades y futuros del Chocó. 2017. http://majestuosoatrato.tierradigna.org/

Navarro-Yashin, Yael. 2012. The Make-Believe Space: Affective Geography in a Postwar Polity. Durham, NC: Duke University Press.

Oslender, Ulrich. 2008. Comunidades negras y espacio en el Pacífico colombiano. Hacia un giro geográfico en el estudio de los movimientos sociales. Bogotá: ICANH.

—. 2016. The Geographies of Social Movements. Durham, NC: Duke University Press.

Riaño, Pilar y Erin Baines. 2012. "Editorial Note”. International Journal of Transitional Justice, número especial "Transitional Justice and the Everyday” 6 (3): 385-393. https://doi. org/10.1093/ijtj/ijs027

Seigworth, Gregory y Melissa Gregg. 2010. "An Inventory of Shimmers". En The Affect Theory Reader, editado por Melissa Gregg y Gregory Seigworth, 1-25. Durham, NC: Duke University Press.

Ulloa, Astrid. 1992. Kipará: dibujo y pintura, dos formas embera de representar el mundo. Bogotá: Universidad Nacional de Colombia.

Velásquez, Rogerio. 2000. Fragmentos de historia, etnografía y narraciones del Pacífico colombiano negro. Bogotá: ICANH.

Vergara-Figueroa, Aurora. 2018. Afrodescendant Resistance to Deracination in Colombia. Massacre at Bellavista-Bojayá-Chocó. Cham (Suiza): Palgrave Macmillan. 